\title{
Editorial
}

Z Gerontol Geriat 2015 - 48:587

DOI 10.1007/s00391-015-0944-6

Online publiziert: 12. August 2015

๑) Springer-Verlag Berlin Heidelberg 2015

CrossMark
Hans Jürgen Heppner ${ }^{1}$ Markus Gosch ${ }^{2}$

${ }^{1}$ Geriatrische Klinik und Tagesklinik, Helios Klinik Schwelm, Universität Witten/Herdecke, Schwelm, Deutschland

${ }^{2}$ Universitätsklinik für Geriatrie, Paracelsus Medizinische Privatuniversität, Nürnberg, Deutschland

\section{Alter und Infektionen}

\section{Seit jeher zwei auf das Engste verknüpfte Themen}

Es gibt tausend Krankheiten, aber nur eine Gesundheit.

(Ludwig Börne, http://www.bk-luebeck.eu/ zitate-boerne.html)

Schon Sir William Osler (1849-1919) erkannte, dass Infektionserkrankungen, allen voran die Pneumonie, todbringende Erkrankungen für den alten Menschen sind.

Pneumonia may well be called the friend of the aged. Taken off by it in an acute, short, not often painful illness, the old man escapes those "cold gradations of decay" so distressing to himself and to his friends.

Er beobachtete, dass die meisten Patienten zwar mit einer Pneumonie, aber nicht nur an der Pneumonie verstarben [1]. So wurden zu dieser Zeit Infektionen, allen voran die Lungenentzündung, noch als erlösend angesehen, doch dies hat sich im Laufe der Jahre deutlich gewandelt, und die Pneumonie, wie viele andere Infektionskrankheiten, ist im Alter erfolgreich behandelbar.

Aktuell stehen akute Infektionen in der Todesursachenstatistik bei älteren Patienten an 6. Stelle [2].

Allerdings stellen sich Symptomatik und Diagnostik in der speziellen Gruppe geriatrischer Patienten unspezifisch und kompliziert dar. Allein die Diagnose einer Harnwegsinfektion bedeutet beim geriatrischen Patienten aufgrund oftmals unklarer klinischer Symptome eine große
Herausforderung (Becher et al.). Einerseits sollten geriatrische Patienten aufgrund ihrer Vulnerabilität möglichst frühzeitig eine antibiotische Therapie erhalten, andererseits bedeutet gerade in dieser Altersgruppe jede unüberlegte antibiotische Therapie ein weiteres Risiko für Komplikationen (Clostridieninfektion, unerwünschte Arzneimittelwirkungen und Interaktionen mit der bestehenden medikamentösen Therapie). Mehrere Beiträge widmen sich dieser Herausforderung. So legen Bertsch et al. in ihrer Übersicht dar, dass sich die Akute-Phase-Reaktion beim alten Menschen anders äußert, und Christ et al. beschreiben die Schwierigkeiten bei der Infektionsdetektion beim alten Menschen in der Notaufnahme. Nach Diagnosestellung ist es wichtig, das Risiko für den Patienten abzuschätzen, und Pflug et al. bewerten die unterschiedlichen Risiko-Scores für den geriatrischen Patienten.

Auch die Prävention darf nicht zu kurz kommen. Kwetkat et al. geben darüber hinaus ein Update über den Stellenwert der Impfungen im Alter.

Mit unserem Themenschwerpunkt wollen wir Ihnen nicht nur einen aktuellen Überblick bieten, sondern Sie auch motivieren, dieses spannende Gebiet mit den vielen offenen Fragen wissenschaftlich zu bearbeiten.

Zuletzt möchten wir Ihnen viel Spaß bei der interessanten Lektüre des Themenschwerpunkts „Infektionen beim alten Menschen" wünschen.
Herzlichst,

Ihre

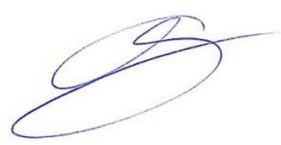

M. Gosch

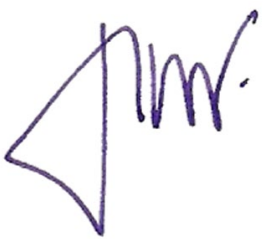

H.J. Heppner

\section{Korrespondenzadresse}

Prof. H. Jürgen Heppner

Geriatrische Klinik und Tagesklinik

Helios Klinik Schwelm, Universität Witten/

Herdecke

Dr.-Moeller-Str. 15, 58332 Schwelm

Hans.Heppner@uni-wh.de

Univ.-Prof. Dr. med. univ. M. Gosch

Universitätsklinik für Geriatrie,

Paracelsus Medizinische Privatuniversität

Klinikum Nürnberg

Prof.-Ernst-Nathan-Str. 1, 90419 Nürnberg

markus.gosch@klinikum-nuernberg.de

Interessenkonflikt. M. Gosch und H.J. Heppner geben an, dass kein Interessenkonflikt besteht.

\section{Literatur}

1. Osler W (1909) The principles and practice of medicine, 7. Aufl. D. Appleton, New York, S 165

2. www.destatis.de. Zugegriffen: 6. Juli 2015 